\title{
LEVEL OF EDUCATION AS A RISK FACTOR FOR EXTENSIVE PREVALENCE OF CERVICAL INTERVERTEBRAL DISC DEGENERATIVE CHANGES AND CHRONIC NECK PAIN
}

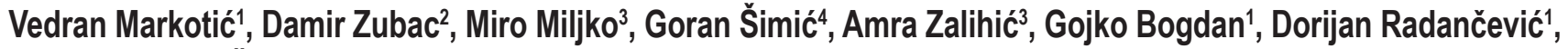 \\ Ana Dugandžić Šimić4, Josip Mašković ${ }^{1}$ \\ 'Department of Clinical Radiology, University Hospital, Mostar, Bosnia and Herzegovina \\ ${ }^{2} S c i e n c e$ and Research Centre Koper, Institute for Kinesiology Research, Koper, Slovenia

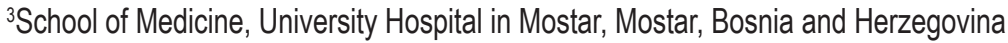 \\ ${ }^{4}$ University Hospital, Mostar, Bosnia and Herzegovina
}

\section{SUMMARY}

Objective: The aim of this study was to document the prevalence of degenerative intervertebral disc changes in the patients who previously reported symptoms of neck pain and to determine the influence of education level on degenerative intervertebral disc changes and subsequent chronic neck pain.

Methods: One hundred and twelve patients were randomly selected from the University Hospital in Mostar, Bosna and Herzegovina, (aged $48.5 \pm 12.7$ years) and submitted to magnetic resonance imaging (MRI) of the cervical spine. MRI of 3.0 T (Siemens, Skyrim, Erlangen, Germany) was used to obtain cervical spine images. Patients were separated into two groups based on their education level: low education level (LLE) and high education level (HLE). Pfirrmann classification was used to document intervertebral disc degeneration, while self-reported chronic neck pain was evaluated using the previously validated Oswestry questionnaire.

Results: The entire logistic regression model containing all predictors was statistically significant, $\left(X^{2}(3)=12.2, p=0.02\right)$, and was able to distinguish between respondents who had chronic neck pain and vice versa. The model explained between 10.0\% (Cox-Snell $R^{2}$ ) and $13.8 \%$ (Nagelkerke $\mathrm{R}^{2}$ ) of common variance with Pfirrmann classification, and it had the strength to discriminate and correctly classify $69.6 \%$ of patients. The probability of a patient being classified in the high or low group of degenerative disc changes according to the Pfirrmann scale was associated with the education level (Wald test: $5.5, p=0.02$ ). Based on the Pfirrmann assessment scale, the HLE group was significantly different from the LLE group in the degree of degenerative changes of the cervical intervertebral discs $(U=1,077.5, p=0.001)$.

Conclusion: A moderate level of intervertebral disc degenerative changes (grade II and III) was equally matched among all patients, while the overall results suggest a higher level of education as a risk factor leading to cervical disc degenerative changes, regardless of age differences among respondents.

Key words: education, disc degeneration, cervical vertebra, chronic neck pain

Address for correspondence: V. Markotić, University Hospital, Department of clinical radiology, Bijeli brijeg b.b., 88000 Mostar, Bosnia and Herzegovina. E-mail:vedranmarkotic@gmail.com

https://doi.org/10.21101/cejph.a4897

\section{INTRODUCTION}

A sedentary lifestyle can include office-related tasks, academic assignments that require prolonged sitting, physical inactivity, and long car rides, which are considered important factors that cause mechanical disorders of the cervical spine structures (CSS) (1). Likewise, sudden movements and poor posture (2) concomitant with weak biomechanical characteristics of the CSS may also lead to the development of cervical disc degenerative changes and subsequent neck pain $(1,2)$. The functional units of the CSS, called vertebral dynamic segments, represent key components in the structure of the cervical spine, commonly affected by degenerative changes of the cervical spine (1). These units consist of two adjacent vertebrae, an intervertebral disc, intervertebral joints, intervertebral apertures (which the blood vessels and nerves pass through), transverse and spinous processes, a spinal canal and ligaments, tendons, connective tissue, and paravertebral muscles (3). Any change in the structure of these components can compromise the function of the intervertebral discs, leading the nucleus to become more compact and stiffer as a consequence of loss in the liquid component, and subsequently to develop fissures and ruptures within the annulus (4-8). Over time, these loads may accumulate and contribute to the development of degenerative changes in the intervertebral discs, resulting in the development of painful neck conditions in the general population (7). For example, extensive degenerative 
changes may promote ankylosis of the dynamic segment that may cause neck pain $(1,6,9)$.

Chronic neck pain and degenerative changes of intervertebral discs of the cervical spine could be defined as part of the condition known in the literature as 'DDD', which stands for degenerative disc disease. This condition affects young adults and middle-aged people who are active and otherwise in good health, although it could be speculated that some populations might be more susceptible to the development of DDD than others $(8,10,19)$. Also, errors in the classifications or subjective mistakes of magnetic resonance imaging (MRI) readings are common among radiologists (11-13), probably due to various classification methods and diagnosis of degenerative intervertebral discs changes used in the past to determine the degree of degenerative intervertebral discs alterations. Pfirrmann et al. (15) published MRI classification guidelines in 2001 in order to overcome on-going classification debate among medical professionals. Indeed, they published a five-item scale to classify the degree of degenerative changes in the intervertebral discs of the lumbar and later of the cervical spine, dependent on different MRI findings of the disc. They reported modest intra- and interobserver kappa coefficients ranging from 0.69 to 90 , respectively, while the overall agreement of the questionnaire items and MRI scan was $84 \%$ in common variance. Thus, the scale was shown to be both valid and sensitive to classify the degree of degenerative disk changes, and it is used in clinical practice $(14,15)$.

A recent article (8) suggests that the prevalence of chronic neck pain tends to increase in the general population; meanwhile, data on degenerative changes of the cervical spine remain scarce. Bogduk et al. (16) suggest that degenerative changes of intervertebral discs originate from poor posture (i.e. straining position of the entire spine is sitting with mild anteflexion) during academic assignments such as reading, writing, or working on the computer. Weidenbaum et al. (17) re-confirmed these findings by documenting images of lesions and pathological and physiological changes of the intervertebral discs using the gold standard, MRI of the spine. According to the applied research of Movahhed et al. (19) chronic neck pain was moderately prevalent (by $41.8 \%$ ) among recently graduated dentists and dental students in Iran, mainly due to extensive practical work coupled with the requirement of longterm standing. Moreover, Movahhed et al. (19) suggest that the higher-educated population is more susceptible to the development of degenerative disc changes than others. According to their study, education level was considered to be a critical factor that contributes to the development of chronic neck pain. However, data on the prevalence of chronic neck pain in the general population have not been reported in the literature. Thus, findings on the prevalence of cervical degenerative disc changes among the general population remain unknown. The aim of this study was twofold: to document the prevalence of chronic neck pain and to examine whether the level of education is a potential factor leading to the development of degenerative disc changes in the cervical spine.

\section{MATERIALS AND METHODS}

\section{Participants}

One hundred and twelve patients (aged $48.5 \pm 12.7$ years) were randomly selected and submitted to MRI of the cervical spine
(Table 2). This represents nearly one third of all patients admitted by both their general physician and neurologist to the Department of Radiology during 15 months of the investigation period (1 June 2014 to 1 November 2015). More precisely, patients who previously reported symptoms of neck pain to their general physician and were admitted by their neurologist to MRI scanning were included in the study. Preliminary medical history analysis excluded patients with tumours, trauma, and autoimmune and inflammatory diseases. After providing informed consent, the participants were assigned to two groups, based on their level of education, as follows: group 1 - high education level (HLE) and group 2 low education level (LLE) group. Basic anthropometric data for both groups are presented in Table 2. All participants were fully informed of any risks and benefits associated with the experiment before any data were collected. The study protocol was approved by the Institutional Ethics Committee of the University Hospital in Mostar and conformed to the standards set by the Declaration of Helsinki (2002) and its amendments. Written informed consent was obtained from each participant upon enrolment.

\section{Procedures}

\section{MRI Imaging}

The study was conducted at the Department of Radiology, University Hospital in Mostar, Bosnia and Herzegovina. MRI examination was performed on an MRI machine of 3.0 T (Siemens, Skyrim, Erlangen, Germany) using the head and neck coil. All participants had a complete MRI of the cervical spine with the head in position of retroflexion. All layers had a thickness of $3 \mathrm{~mm}$, and the spacing between the layers (distance factor) was $10 \%$ with a field width of $220 \mathrm{~mm}$. Sequences for the sagittal and axial sections were $\mathrm{T} 1$ tse - sag, T1 tirm - sag, T2 me2d - axi, and T1 tse - axi.

\section{Pfirrmann and Oswestry Classification}

The degree of intervertebral disc degeneration was assessed using the Pfirrmann classification (15): in the first stage the disc is a homogeneous, high-intensity signal and normal height; the second degree includes the inhomogeneous structure of the disc, high-intensity signal with possible horizontal gray lines, clearly delineated boundaries of the annulus and the nucleus, and normal height of the disc; the third degree is characterized by the inhomogeneous structure of the disc with zones of middle intensity. The limit of the annulus and nucleus is unclear, and the height of the disc is normal or discreetly reduced; in the fourth degree the inhomogeneous structure of the intervertebral disc with areas of low signal intensity is seen, with loss of annulus and nucleus limits and moderately reduced height of the intervertebral disc; the fifth degree is characterized by the inhomogeneous structure of the disc, a low-intensity signal, completely vanished limits of the annulus and the nucleus, and an extremely reduced height of the disc (Table 1).

After a general health examination, participants filled out a questionnaire, which was separated into two parts. The first part of the questionnaire consisted of a validated Oswestry questionnaire addressing chronic neck pain $(20,21)$. This questionnaire assessed the limitations of daily activities of patients (previously included in the present study) suffering from 
Table 1. Classification of disc degeneration based on Pfirrmann guidelines (15)

\begin{tabular}{|l|l|l|l|l|}
\hline Grade & Disk structure & $\begin{array}{l}\text { Distinction between nucleus } \\
\text { and annulus }\end{array}$ & Signal intensity & Height of intervertebral disk \\
\hline I & Homogeneous, white & Clear & $\begin{array}{l}\text { Hyperintense, isointense to } \\
\text { cerebrospinal fluid }\end{array}$ & Normal \\
\hline II & $\begin{array}{l}\text { Inhomogeneous, with or without } \\
\text { horizontal bands }\end{array}$ & Clear & $\begin{array}{l}\text { Hyperintense, isointense to } \\
\text { cerebrospinal fluid }\end{array}$ & Normal \\
\hline III & Inhomogeneous, grey & Unclear & Intermediate & Normal to slightly decreased \\
\hline IV & Inhomogeneous, grey to black & Lost & Intermediate to hypointense & Normal to moderately decreased \\
\hline V & Inhomogeneous, black & Lost & Hypointense & Collapsed disc space \\
\hline
\end{tabular}

chronic neck pain. Throughout the questionnaire, participants were asked to provide anamnesis data on the severity of pain, personal hygiene, heavy lifting, reading abilities, headaches, overall concentration, occupation, driving, sleeping habits, and recreation. The second part of the questionnaire contained items on socio-demographic data, such as gender, age, height, weight, level of education, duration of education after completing high school, and current occupation. The reliability of the questionnaire was confirmed throughout test-retest correlation (for ordinal variables such as age) and the analysis of equally answered questions (for ordinal and nominal variables). Furthermore, prior to the main study, 30 participants were tested on two occasions, separated by 10 days.

To attenuate the risk of bias, items on the Pfirrmann scale were evaluated by two independent reviewers (radiologist). Agreement between two reviewers was assessed using Cohen's kappa (k statistics); when there was a disagreement between the two reviewers, a third investigator made the final decision on the item in question.

\section{Statistical Analysis}

Statistica v.13.0 (Stat Soft, Tulsa, OK, USA) was used to analyse the data, and the Kolmogorov-Smirnov test was applied to examine the normality of the distribution. Frequencies and counts were calculated for the questionnaire items based on classification data. Non-parametric tests were conducted for any non-normally distributed variables by means of the MannWhitney $\mathrm{U}$ test to determine the differences between groups for all dependent variables.

A binary logistic regression was applied to determine the relation of independent variables in cervical disc degenerative changes. The independent variables (predictors) were smoking, education level, and body mass index (BMI). For needs of logistic regression, the answers concerning cervical disc degenerative changes degree (dependent variable) were clustered into two groups according to the following criteria: 0 , mild degenerative changes, 1st-2nd degree; and 1, advanced degenerative changes, 3rd-5th degree. Logistic regression (binary model) determined partial contributions of four independent variables to the sever-

Table 2. Basic anthropometric characteristics

\begin{tabular}{|l|c|c|c|}
\hline & All & LLE & HLE \\
\hline $\mathrm{N}(\%$ men) & $112(44 \%$ males $)$ & $26(54 \%$ males $)$ & $23(47 \%$ males $)$ \\
\hline Age (years) & $48.5 \pm 12.7$ & $49.5 \pm 13.5$ & $47.5 \pm 11.6$ \\
\hline Body heigth $(\mathrm{m})$ & $1.73 \pm 0.1$ & $1.73 \pm 0.1$ & $1.74 \pm 0.09$ \\
\hline Body mass $(\mathrm{kg})$ & $77.2 \pm 14.7$ & $76.9 \pm 13.6$ & $77.5 \pm 14.8$ \\
\hline BMl & $25.6 \pm 14.1$ & $25.7 \pm 13.9$ & $25.4 \pm 13.7$ \\
\hline
\end{tabular}

LLE - lower level of education; HLE - higher level of education; BMI - body mass index; significantly different at $\mathrm{p}<0.05$

Table 3. Prevalence of degenerative disc change of cervical spine

\begin{tabular}{|l|c|c|c|c|c|c|}
\hline \multirow{2}{*}{} & \multicolumn{2}{|c|}{ All } & \multicolumn{2}{c|}{ LLE } & \multicolumn{2}{c|}{ HLE } \\
\cline { 2 - 7 } & Pfirmann & Oswestry & Pfirrmann & Oswestry & Pfirrmann & Oswestry \\
\hline 1st degree & $17(44 \%)$ & $7(6.1 \%)$ & $14(22 \%)$ & $4(6.3 \%)$ & $3(5.8 \%)$ & $3(5.7 \%)$ \\
\hline 2nd degree & $34(29.6 \%)$ & $27(23.4 \%)$ & $21(33 \%)$ & $18(28.6 \%)$ & $13(25 \%)$ & $9(17.3 \%)$ \\
\hline 3rd degree & $39(33.9 \%)$ & $42(36.5 \%)$ & $21(33 \%)$ & $25(39.7 \%)$ & $18(34.6 \%)$ & $17(32.7 \%)$ \\
\hline 4th degree & $16(13.9 \%)$ & $28(24.3 \%)$ & $12(13.1 \%)$ & $11(13.1 \%)$ & $14(26.9 \%)$ & $17(32.7 \%)$ \\
\hline 5th degree & $9(7.8 \%)$ & $11(9.5 \%)$ & $5(7.9 \%)$ & $5(7.5 \%)$ & $4(7.6 \%)$ & $6(11.5 \%)$ \\
\hline
\end{tabular}

LLE - low education level; HLE - high education level; 1st degree - disc is a homogeneous, high signal intensity and normal height; 2nd degree - inhomogeneous structure of the disc, high signal intensity with possible horizontal gray lines, clearly delineated boundaries of the annulus and the nucleus, and normal height of the disc; 3 rd degree - inhomogeneous structure of the disc with zones of middle intensity. The limit of the annulus and nucleus is unclear, and height of the disc is normal or discreetly reduced; 4th degree - inhomogeneous structure of the intervertebral disc with areas of low signal intensity, loss of annulus and nucleus limits and moderately reduced height of the intervertebral disc; 5th degree - inhomogeneous structure of the disc, low-intensity signal, limit of the annulus and the nucleus completely vanished and the height of the disc is extremely reduced. 
ity of degenerative changes in the cervical spine (criteria). Data were presented by mean \pm standard deviation when appropriate. Statistical significance was accepted at $\mathrm{p}<0.05$.

\section{RESULTS}

\section{Reliability Issues}

Inter-rater reliability showed a high level of agreement between the two independent raters, $\mathrm{k}$ statistic $=0.90$. Test-retest reliability for the items on the questionnaire document: Spearman correlation ranged from 0.95 to 0.99 (for ordinal variables). The consistency of the test-retest answers was 95 to $100 \%$ (for nominal variables), proving reliability of the questionnaire. Approximately $23 \%$ of participants self-reported habitual smoking, while only $3 \%$ self-reported excessive physical activity on a daily basis. There were no significant differences between the LLE and HLE groups in the aforementioned dependent variables $(p=0.09$; $\mathrm{p}=0.91$, respectively).

\section{Demographic Data}

Table 2 presents the demographic data and basic anthropometric characteristics of the subjects.

\section{Prevalence of Degenerative Disc Changes of the Cervical Spine}

Table 3 documents the third degree as the most abundant pattern of the degenerative changes by $33.9 \%$ of the intervertebral discs of the cervical spine among all participants. In addition, the most frequently self-reported symptom on the Oswestry scale was the third degree as well (by $36.5 \%$ ). In general, symptoms associated with the CSS were equally prevalent among LLE and HLE participants.

\section{Binary Logistic Regression}

As shown in Table 4, binary logistical regression was performed to address the association between independent variables and the binary high or low Pfirmann scale values. The model contained three independent variables (BMI, smoking habits, and physical activity). The entire model containing all predictors was statistically significant, $\left(\chi^{2}(3)=12.2, p=0.02\right)$, indicating the ability to distinguish between respondents who had cervical disc degenerative changes and those who did not. The model as a whole explained between $10.0 \%$ (Cox-Snell R squared) and $13.8 \%$ (Nagelkerke $\mathrm{R}^{2}$ ) of the common variance in cervical disc degenerative changes, and it had the strength to discriminate and correctly classify $69.6 \%$ of cases as the probability of a patient being classified in the high or low group of cervical disc degenerative changes, which, according to Pfirrmann, was associated with the education level (Wald test: $5.5, \mathrm{p}=0.02$ ). Finally, the Hosmer-Lemeshow test was not significant, indicating goodness of fit for this model $\left(\chi^{2}=3.9, p=0.87\right)$.

There were no significant differences between the LLE and HLE groups in self-reported symptoms associated with the CSS (Oswestry scale; $U=1,439, p=0.24$ ) (Fig. 1). On the contrary, based on the Pfirrmann assessment scale, the HLE group significantly differed from the LLE group in the degree of degenerative changes in intervertebral discs of the cervical spine $(\mathrm{U}=1,077.5, \mathrm{p}=0.001)$.

Table 4. Binary logistic regression

\begin{tabular}{|c|c|c|c|c|c|c|c|c|}
\hline & \multirow{2}{*}{ B } & \multirow{2}{*}{ SE } & \multirow{2}{*}{ Wald } & \multirow{2}{*}{$d f$} & \multirow{2}{*}{$p$-value } & \multirow{2}{*}{$\operatorname{Exp}(B)$} & \multicolumn{2}{|c|}{$95 \% \mathrm{Cl}$ for $\operatorname{Exp}(\mathrm{B})$} \\
\hline & & & & & & & Lower & Upper \\
\hline Smoking & -0.69 & 0.51 & 1.84 & 1 & 0.17 & 0.50 & 0.18 & 1.10 \\
\hline Education level & -0.97 & 0.41 & 5.50 & 1 & $0.02^{*}$ & 2.63 & 1.17 & 5.93 \\
\hline BMI & -0.02 & 0.05 & 0.18 & 1 & 0.67 & 0.97 & 0.87 & 1.10 \\
\hline Age & -0.03 & 0.02 & 2.65 & 1 & 0.13 & 0.97 & 0.94 & 1.00 \\
\hline Constant & 2.21 & 2.06 & 1.15 & 1 & 0.28 & 9.20 & & \\
\hline
\end{tabular}

B - Beta coefficient; BMl - body mass index; df - degrees of freedom; 95\% Cl - 95\% Cl confidence intervals; *statistical significance $\mathrm{p}<0.05$

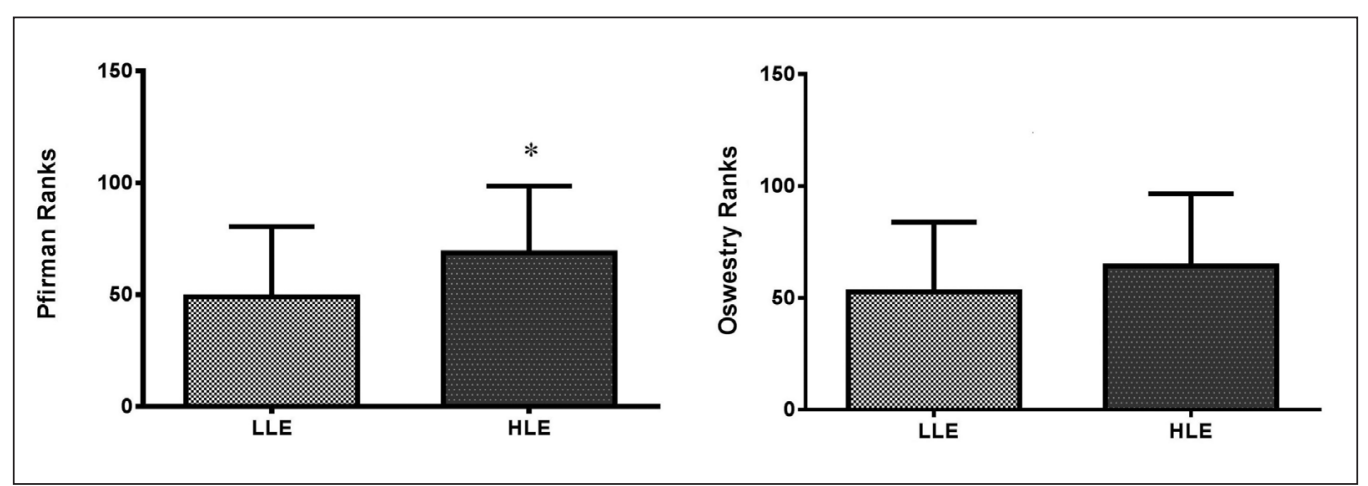

Fig. 1. The difference between HLE and LLE groups in the Pfirman classification and Oswestry questionnaire. HLE - High education level; LLE - low education level; *different from LLE; $p<0.05$ 


\section{DISCUSSION}

This study involved extensive MRI research to address the prevalence of cervical disc degenerative changes in the general population, who previously reported symptoms of neck pain to their general physician. The main findings of the present investigation were as follows: regardless of the education level, 55\% of all patients had moderate cervical disc degenerative changes. The aforementioned changes were defined as a part of the condition known in the literature as 'DDD', which represents a clinical condition manifested as degenerative disc disease, according to the Pfirrmann classification scale, the level of education was recognised as a risk factor leading to the development of cervical disc degenerative changes, the HLE group differed from the LLE group in the cervical disc degenerative changes by a degree $(\mathrm{U}=1,077.5, \mathrm{p}=0.001)$.

The prevalence of overall cervical disc degenerative changes was $\sim 55 \%$, equally matched among low and moderate CSS alterations (i.e. 2nd and 3rd degree), while a twofold greater difference was noted in the 4th degree (Table 3 ). The results were not consistent with the findings of Lee et al. (18), who found $95.4 \%$ of cervical disc degenerative changes in 102 patients. The possible underlying cause of the discrepancy between the data presented in this paper and the study of Lee et al. (18) was the fact that this study observed cervical disc degenerative changes, whereas Lee and authors (18) observed the degenerative changes of the overall CSS. Although some authors have speculated that certain populations are more susceptible to the development of cervical disc degenerative changes or DDD than others, this study indirectly confirms such a postulate by demonstrating differences in Pfirrmann classification among two groups of patients (Fig. 1). Moreover, cervical spine degenerative changes were not exclusively age-related. More precisely, young adults and middleaged people previously physically active and otherwise in good health were equally affected by the development of cervical disc degenerative changes (Table 3).

According to the binary logistic regression (Table 4), HLE was established as an important risk factor leading to the development of cervical disc degenerative changes, according to Wald test data $(\mathrm{W}=5.5, \mathrm{p}=0.02)$. Notably, smoking, $\mathrm{BMI}$, and vigorous physical exercise were not related to the prevalence of cervical disc degenerative changes or neck pain. Thus, the findings of the present study, which considers education level as a risk factor for cervical disc degenerative changes, were consistent with the study of Movahhed et al. (19), who reported $42 \%$ of chronic neck pain among Iranian dentists and dental students. The authors assumed that a high education level and practical work play a role in the underlying mechanisms of CCS. However, Movahhed et al. (19) did not include a control group in their study. In contrast, this study divided patients according to their education level and found differences between the LLE and HLE $(U=1,077.5, p=0.001)$ groups, further supporting the aforementioned postulations based on logistic regression findings. Thus, the education level was instrumental in cervical disc degenerative change patterns between the third and fourth levels of the Pfirrmann classification scale. Nevertheless, discrepancies in the methodological approach between this and similar studies represent important methodological prerequisites and preclude a conclusive position within current health-hazard population characterisation.
However, regardless of the overall differences established between LLE and HLE (Fig. 1), the occurrence of cervical disc degenerative changes in relation to the education level of patients was twofold greater in the fourth degree (i.e. severe changes), but not in the third degree (i.e. moderate changes) (Table 3), according to Pfirrmann classification. Regarding the latter, only $\sim 13 \%$ of LLE patients were characterised by a severe condition of disc degeneration; on the other hand, $27 \%$ of HLE patients had severe cervical disc degenerative changes. Thus, severe rather than moderate cervical disc degenerative changes were more pronounced and related to education level. Apparently, the education level was not the driving force behind low and moderate cervical disc degenerative changes, and the patterns of severe degenerative changes became more evident as the level of education increased.

Finally, the patients who achieved a low score on the Oswestry test were not candidates for early MRI, and this may be translated into a decrease of MRI examinations, from a practical standpoint. Needless to say, using Oswestry classification would contribute to saving time and financial resources by applying the Pfirrmann classification in everyday practice, as demonstrated in our study, and it would be an applicable diagnostic tool distinguishing different levels of cervical intervertebral disc changes.

\section{CONCLUSION}

Moderate degenerative changes in the cervical spine (II and III level) were equally prevalent among all respondents, regardless of education level, whereas the overall results indicate a higher education level as a risk factor for developing cervical disc degenerative changes. Thus, education level plays a negligible role in low and moderate cervical disc degenerative changes, while the patterns of severe degenerative changes become more pronounced as the level of education increases, as changes become more pronounced concomitant with HLE. Data from Oswestry classification analysis show that there is no statistically significant relationship between different levels of education and chronic neck pain.

\section{REFERENCES}

1. Adams MA, Freeman BJ, Morrison HP, Nelson IW, Dolan P. Mechanical initiation of intervertebral disc degeneration. Spine (Phila Pa 1976). 2000 Jul 1;25(13):1625-36.

2. Pećina M. Degenerative joint disease. In: Pećina M, et al. Orthopedia. Zagreb: Naklada Ljevak; 2000. p. 99-105. (In Croatian.)

3. Krmpotić-Nemanić J, Marušić A. Human anatomy. Zagreb: Medicinska Naklada; 2001. p. 42-4. (In Croatian.)

4. Pećina M. Degenerative joint disease. In: Gamulin S, Marušić M, Krvavica S. Pathophysiology. Zagreb: Medicinska Naklada; 1996. p. 529-31. (In Croatian.)

5. Hoenen-Clavert V, Rat AC, Loeuille D, Bettembourg-Brault I, MichelBatot C, Blum A, et al. Inflammatory and structural evaluation in spondyloarthritis: magnetic resonance imaging analysis of axial and peripheral involvement. J Rheumatol. 2007 Apr;34(4):762-8.

6. Karchevsky M, Schweitzer ME, Carrino JA, Zoga A, Montgomery D, Parker L. Reactive endplate marrow changes: a systematic morphologic and epidemiologic evaluation. Skeletal Radiol. 2005 Mar;34(3):125-9.

7. Urban JP, Roberts S. Degeneration of the intervertebral disc. Arthritis Res Ther. 2003;5(3):120-30.

8. Bogduk N. Degenerative joint disease of the spine. Radiol Clin North Am. 2012 Jul;50(4):613-28. 
9. Peterson CK, Humphreys BK, Pringle TC. Prevalence of modic degenerative marrow changes in the cervical spine. J Manipulative Physiol Ther. 2007 Jan;30(1):5-10.

10. Simmons ED Jr, Guntupalli M, Kowalski JM, Braun F, Seidel T. Familial predisposition for degenerative disc disease. A case-control study. Spine (Phila Pa 1976). 1996 Jul 1;21(13):1527-9.

11. Jarosz J, Bingham JB, Pemberton J, Sambrook PN, Spector TD. An atlas for scoring cervical and lumbar disc degeneration. London: Springer Verlag; 1997.

12. Raininko R, Manninen H, Battié MC, Gibbons LE, Gill K, Fisher LD. Observer variability in the assessment of disc degeneration on magnetic resonance images of the lumbar and thoracic spine. Spine (Phila Pa 1976). 1995 May 1;20(9):1029-35.

13. Brant-Zawadzki MN, Jensen MC, Obuchowski N, Ross JS, Modic MT. Interobserver and intraobserver variability in interpretation of lumbar disc abnormalities. A comparison of two nomenclatures. Spine (Phila Pa 1976). 1995 Jun 1;20(11):1257-63; discussion 1264.

14. Griffiths GO, Sambrook PN, Jarosz J, Bingham J, Spector TD. Development of an atlas for grading cervical and lumbar discpathology by magnetic resonance imaging. Br J Rheumatol. 1997;36 Suppl 1:95.

15. Pfirrmann CW, Metzdorf A, Zanetti M, Hodler J, Boos N. Magnetic resonance classification of lumbar intervertebral disc degeneration. Spine (Phila Pa 1976). 2001 Sep 1;26(17):1873-8.
16. Bogduk N, Mercer S. Biomechanics of the cervical spine. I: normal kinematics. Clin Biomech (Bristol, Avon). 2000 Nov;15(9):633-48.

17. Weidenbaum M, Foster RJ, Best BA, Saed-Nejad F, Nickoloff E, Newhouse $\mathrm{J}$, et al. Correlating magnetic resonance imaging with the biochemical content of the normal human intervertebral disc. J Orthop Res. 1992 Jul;10(4):552-61.

18. Lee TH, Kim SJ, Lim SM. Prevalence of disc degeneration in asymptomatic Korean subjects. Part 2: cervical spine. J Korean Neurosurg Soc. 2013 Feb;53(2):89-95.

19. Movahhed T, Ajami B, Soltani M, Shakeri MT, Dehghani M. Musculoskeletal pain reports among Mashhad dental students, Iran. Pak J Biol Sci. 2013 Jan 15;16(2):80-5

20. Wheeler AH, Goolkasian P, Baird AC, Darden BV 2nd. Development of the Neck Pain and Disability Scale. Item analysis, face, and criterionrelated validity. Spine (Phila Pa 1976). 1999 Jul 1;24(13):1290-4.

21. Soysal M, Kara B, Arda MN. Assessment of physical activity in patients with chronic low back or neck pain. Turk Neurosurg. 2013;23(1):75-80.

Received August 8, 2016 Accepted in revised form November 23, 2016 